International Journal of Pure and Applied Mathematics

Volume 95 No. $4 \quad 2014,629-634$

ISSN: 1311-8080 (printed version); ISSN: 1314-3395 (on-line version)

url: http://www.ijpam.eu

doi: http://dx.doi.org/10.12732/ijpam.v95i4.16

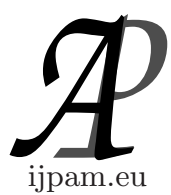

\title{
SYMMETRIC TENSOR RANK WITH RESPECT TO CURVES
}

\author{
E. Ballico \\ Department of Mathematics \\ University of Trento \\ 38123 Povo (Trento) - Via Sommarive, 14, ITALY
}

\begin{abstract}
Let $\nu: d: \mathbb{P}^{m} \rightarrow \mathbb{P}^{r}, r:=\left(\begin{array}{c}m+d \\ m\end{array}\right)-1$, be the order $d$ Veronese embedding. For any $P \in \mathbb{P}^{r}$ let $c(P)$ (resp. $c c(P)$, resp. $i c(P)$ ) be the minimal degree of a reduced (resp. reduced and connected, resp. integral) curve $C \subset \mathbb{P}^{m}$ such that $P \in\left\langle\nu_{d}(C)\right\rangle$. We study these invariants when $P$ has border rank $\leq 4$.
\end{abstract}

AMS Subject Classification: $14 \mathrm{~N} 05$

Key Words: symmetric tensor rank, irreducible curve, border rank

\section{Ranks with Respect to Curves}

Let $X \subset \mathbb{P}^{r}$ be an integral and non-degenerate variety of dimension $m \geq 2$. For each $P \in \mathbb{P}^{r}$ let $c_{X}(P)$ (resp. $c c_{X}(P)$, resp. $i c_{X}(P)$ ) be the minimal degree of a reduced (resp. reduced and connected, resp. integral) curve $C \subset X$ such that $P \in\langle C\rangle$, where \langle\rangle denote the linear span). Obvious examples shows that it is better to consider reduced curves, not just locally Cohen-Macaulay curves. Obviously $i c_{X}(P) \geq c c_{X}(P) \geq c_{X}(P)$ and $c_{X}(P)$ is at least the minimal de- 
gree of an integral curve contained in $X$. However in the most important case of $X$-rank, the symmetric tensor, this is integer we are looking at, but rather these number divided by $d$. Fix integers $m \geq 1$ and $d \geq 1$. Let $\nu_{d}: \mathbb{P}^{m} \rightarrow \mathbb{P}^{r}$, $r:=\left(\begin{array}{c}m+d \\ m\end{array}\right)-1$, denote the order $d$ Veronese embedded of $\mathbb{P}^{m}$, i.e. the embedding of $\mathbb{P}^{m}$ by the complete linear system. Set $X_{m, d}:=\nu_{d}\left(\mathbb{P}^{m}\right)$. Now assume $m \geq 2$. For any $P \in \mathbb{P}^{r}$ set $c_{m, d}(P):=c_{X_{m, d}}(P) / d, c c_{m, d}(P):=c c_{X_{m, d}}(P) / d$, and $i c_{m, d}(P):=i c_{X_{m, d}}(P) / d$ The integer $c_{m, d}(P)\left(\right.$ resp. $c c_{m, d}(P)$, resp. $\left.i c_{m, d}(P)\right)$ is the minimal degree of a reduced (resp. reduced and connected, resp. integral) curve $C \subset \mathbb{P}^{m}$ such that $P \in\langle\nu(C)\rangle$. We often write $c(P), c c(P)$ and $i c(P)$ instead of $c_{m, d}(P), c c_{m, d}(P)$ and $i c_{m, d}(P)$, respectively, and call $r(P)$ the symmetric tensor rank of $P, b(P)$ its border rank and $z(P)$ its cactus rank (also called the scheme-rank) (we may drop the subscript $m$, because if $P$ depends from $n<m$ variables, then $b_{n, d}(P)=b_{m, d}(P)$ (see [7, Proposition 2.1] for the tensor rank and the border tensor rank). If $m=2$, then $c c(P)=c(P)$ for all $P$, because any plane curve is connected. Let $G c(m, d)$ (resp. $G c c(m, d)$, resp. $\operatorname{Gic}(m, d))$ be the integer $c_{m, d}(P)$ (resp. $c c_{m, d}(P)$, resp. $\left.i c_{m, d}(P)\right)$ for a general $P \in \mathbb{P}^{r}$. Let $M c(m, d)(\operatorname{resp} . M c c(m, d)$, resp. $\operatorname{Mic}(m, d))$ be the maximal integer $c_{m, d}(P)$ (resp. $c c_{m, d}(P)$, resp. $\left.i c_{m, d}(P)\right)$ for some $P \in \mathbb{P}^{r}$.

Obviously $c(P)=1 \Longleftrightarrow c c(P)=1 \Longleftrightarrow c c(P)=1 \Longleftrightarrow$ " there is a line $L \subseteq \mathbb{P}^{m}$ such that $P \in\left\langle\nu_{d}(L)\right\rangle "$.

In this note we compute all integers $c(P), c c(P)$ and $i c(P)$ when $P$ has border rank $\leq 4$ (Propositions 2, 3,4 and 5). Following [4] we also make the following observation.

Proposition 1. We have $M c(m, d) \leq 2 \cdot G c(m, d)$.

Proof. There is a non-empty open subset $U \subset \mathbb{P}^{r}$ such that $c_{m, d}(O)=$ $G c(m, d)$ for all $O \in U$. Fix $P \in \mathbb{P}^{r}$ and take a general line $L \subset \mathbb{P}^{r}$ though $P$. Since $L \cap U \neq \emptyset$, there are $P_{1}, P_{2} \in U \cap L$ such that $P_{1} \neq P_{2}$. Take $C_{i} \subset \mathbb{P}^{m}$, $i=1,2$,

Remark 1. Fix an integer $t \geq 1$. Instead of curves in the definition of $c_{m, d}(P)$ we may take finite unions of curves of degree $\leq t$ (in the case $t=1$ we use finite unions of lines, the so-called stick-figures). We call $\operatorname{lr}(P)$ the minimal degree of a union $E \subset \mathbb{P}^{m}$ of lines with $P \in\left\langle\nu_{d}(E)\right\rangle$ (we write $l \operatorname{cr}(P)$ if we add the condition that $E$ is connected). Proposition 1 holds is for these invariants (if we don't assume the connectedness).

Proposition 2. All points of border rank $\leq 2$ have $i c(P)=c c(P)=$ $c(P)=1$. 
Proof. The case $b=1$ is obvious, because every point of $\mathbb{P}^{m}$ is contained in a line. Now assume $b=2$. If $r(P)=2$, then $i c(P)=c(P)=c c(P)$, because any two points of $\mathbb{P} m$ are contained in a line. If $r(P) \neq 2$, the proof of $[3$, Theorem 32] gives the existence of a line $L \subset \mathbb{P}^{m}$ such that $P \in\left\langle\nu_{d}(L)\right\rangle$ and hence $i c(P)=c(P)=c c(P)$.

Proposition 3. Assume $m \geq 2, d \geq 4, b(P)=3$ and that $P \notin\left\langle\nu_{d}(L)\right\rangle$ for any line $L \subset \mathbb{P}^{2}$. Then $c(P)=c c(P)=i c(P)=2$.

Proof. Since $P \notin\left\langle\nu_{d}(L)\right\rangle$ for any line, we have $c(P)>1$. Since $d \geq b(P)-1$ there is a degree 3 zero-dimensional scheme $Z \subset \mathbb{P}^{3}$ such that $P \in\left\langle\nu_{d}(Z)\right\rangle([6$, Lemma 2.6], [5, Proposition 2.5]). We have $Z \neq 2 O$ for some $O \in \mathbb{P}^{m}$, because $b(P)>2([3$, proof of Theorem 37]). By assumption $Z$ is not contained in a line. Hence it is elementary to check that it is contained in a smooth conic $C$. Since $\left\langle\nu_{d}(C)\right\rangle \supseteq\left\langle\nu_{d}(Z)\right\rangle$, we get $i c(P)=2$ and hence $c(P)=c c(P)=2$.

Proposition 4. Assume $m=2, d \geq 7$ and take $P$ with border rank 4 and with $P \notin\left\langle\nu_{d}(L)\right\rangle$ for any line $L \subset \mathbb{P}^{2}$. Then $c c(P)=c(P)=2$. We have $i c(P)=1$ if $r(P)=d-2$ and for some (but not all) points $P$ with $r(P)=4$. We have $i c(P) \leq 3$ and $i c(P)=3$ only if either there is a line $L \subset \mathbb{P}^{2}$ with $\operatorname{deg}(Z \cap L)=3$ or $Z$ is the the complete intersection of two conics through some $O \in \mathbb{P}^{2}$ and $Z \supset 2 O$.

Proof. The assumption " $P \notin\left\langle\nu_{d}(L)\right\rangle$ for any line $L \subset \mathbb{P}^{2}$ " gives $c(P)>1$. Since $m=2$, we have $c c(P)=c(P)$. Since $d \geq b(P)-1$, there is a zerodimensional scheme $Z \subset \mathbb{P}^{2}$ with $\operatorname{deg}(Z)=4$ such that $P \in\langle\nu(Z)\rangle([6$, Lemma 2.6], [5, Proposition 2.5]). Every zero-dimensional scheme of $\mathbb{P}^{2}$ is smoothable. Since $d \geq 2 b(P)-1$, the scheme $Z$ evincing $b(P)$ is unique (proof as in $[6$, Theorem 1.8] which consider the case of reduced schemes, using [3, Lemma 34], which is stated for arbitrary zero-dimensional schemes).

(i) Assume $i c(P)=2$, i.e. assume the existence of a smooth conic $C$ such that $P \in\left\langle\nu_{d}(C)\right\rangle$, and assume $r(P) \geq d+3$. By [2, case (e) of Theorem 1.2] we have $r(P)=2 d-2$. The curve $\nu_{d}(C)$ is a rational normal curve of degree $2 d$ in its linear span. Call $r^{\prime}$ and $b^{\prime}$ the rank and the border rank of $P$ with respect to $\nu_{d}(C)$. We have $r^{\prime} \geq r$ and $b^{\prime} \geq b$. A theorem of Sylvester gives that $b^{\prime} \leq d+1$ and that either $r^{\prime}=b^{\prime}$ or $r^{\prime}+b^{\prime}=2 d+2([9$, Theorem 4.1], [3, Theorem 23]). Since $r>d+1$ and $b=4$, we get $b^{\prime}=4$ and $r^{\prime}=2 d-2$. Let $Z^{\prime} \subset C$ the degree 4 scheme evincing $b^{\prime}$. Since $d \geq 7$, the uniqueness of $Z$ gives $Z^{\prime}=Z$. Hence $Z \subset C$. Since $C$ is a smooth conic, we get that $Z$ is curvilinear and that $\operatorname{deg}(Z \cap L) \leq 2$ for each line $L$. 
(ii) Assume $r(P)=4$, i.e. assume that $Z$ is formed by 4 non-collinear points. If 3 of these points are contained in a line, then $c(P)=c c(P)=2$, and $i c(P) \leq 3$, because $Z$ is contained in a smooth plane cubic. Step (i) gives $i c(P)>2$ and hence $i c(P)=3$. If no 3 of the points of $Z$ are contained in a line, then $Z$ is contained in a smooth conic and hence $i c(P)=2$.

(iii) We have $h^{0}\left(\mathcal{I}_{Z}(2)\right) \geq 2$. Since we work in characteristic $\neq 2$, no pencil of conics is formed only by double lines. Hence $c(P) \leq 2$. Since $c(P)>1$, in all cases we have $c(P)=2$. By step (i) we have $i c(2)>2$ only if $r(P) \leq d+2$. By step (ii) we may assume $r(P)>4$. $Z$ is a complete intersection of two conics if and only if there is no line $L \subset \mathbb{P}^{2}$ with $\operatorname{deg}(Z \cap L)=3$. If $Z$ is a complete intersection of two conics, then it is contained in a smooth conic if and only if it is curvilinear. If $Z$ is a complete intersection of two conics, then $Z$ is not curvilinear if and only if it contains $2 O$ for some $O \in \mathbb{P}^{2}$.

(iv) Assume that $Z$ is a complete intersection of two conics and that $Z \supset 2 O$ for some $O \in \mathbb{P}^{2}$. Since $h^{1}\left(\mathcal{I}_{Z}(2)\right)=0$, the homogeneous ideal of $Z$ is generated in degree $\leq 3$. Since $Z$ is contained in an irreducible curve of large degree (e.g. for an arbitrary scheme with $Z_{\text {red }}=\{O\}$ take any irreducible curve of $\operatorname{degree} \operatorname{deg}(Z)+2$ with multiplicity $\operatorname{deg}(Z)+1$ at $O$ ), we get that $Z$ is contained in an irreducible cubic. Hence $i c(P)=3$.

(v) Assume that $Z$ is not a complete intersection of two conics. Since $Z$ is not contained in a line, there is a line $L \subset \mathbb{P}^{2}$ such that $\operatorname{deg}(L \cap Z)=3$. As in step (i) we get $i c(P)>2$. The residual scheme $\operatorname{Res}_{L}(Z)$ of $Z$ with respect to $L$ has a degree one and hence it is a point, $O$. We have $h^{1}\left(L, \mathcal{I}_{Z \cap L}(2)\right)=0$. Since $h^{1}\left(\mathcal{I}_{O}(1)\right)=0$, the residual exact sequence (also called the Castelnuovo's sequence) gives $h^{1}\left(\mathcal{I}_{Z}(2)\right)=0$. As in step (iv) we get $i c(P)=3$.

Proposition 5. Assume $d \geq 7, m \geq 3$ and fix $P$ with $b(P)=4$ and $P \notin\langle\nu(H)\rangle$ for any plane $H$. Let $Z$ be the only zero-dimensional scheme evincing the border rank of $P$. We have $2 \leq c(P) \leq i c(P)=c c(P)=3$. We have $c(P)=2$ if and only if either $Z$ has at least 3 connected components or $Z$ has two connected components of degree 2.

Proof. Since $d \geq 7$, there is a unique zero-dimensional scheme $Z$ with $\operatorname{deg}(Z)=4$ and evincing the border rank of $P([6$, Lemma 2.6], [5, Proposition 2.5]). The possible $Z$ 's are classified in [2, Subection 5.3] and they are all curvilinear. Since $P \notin\langle\nu(H)\rangle$ for any plane, we have $c(P) \geq 2, c c(P) \geq 3$, $i c(P) \geq 3, c(P)=2$ if and only if $P \in\left\langle\nu_{d}(E)\right\rangle$ with $E \subset \mathbb{P}^{3}$ the union of two disjoint lines, and $i c(P)=3$ if and only if $P \in\left\langle\nu_{d}(C)\right\rangle$ with $C \subset \mathbb{P}^{3}$ a rational normal curve. Since $P \notin\langle\nu(H)\rangle$ for any $H$ a plane, $Z$ spans $\mathbb{P}^{3}$ and (having degree 4$)$ it is in linearly general position.It is easy to check that every degree 4 
curvilinear scheme in linearly general position is contained in a rational normal curve (when $Z=Z^{\prime} \sqcup\{O\}$ with $\operatorname{deg}\left(Z^{\prime}\right)=3$ one may use [1]; in the general case one can use [8, Theorem 3.2]).

Since $\langle Z\rangle=\mathbb{P}^{3}, Z$ is contained in a disjoint union of 2 lines if and only if either $Z$ has at least 3 connected components or it has two connected components of degree two. In these cases we have $c(P)=2$.

Now assume $Z=Z^{\prime} \sqcup\{O\}$ with $Z^{\prime}$ connected of degree 3 and $O$ a point with $O \notin\left\langle Z^{\prime}\right\rangle$. In this case we have $r(P)=2 d([2$, Proposition 5.22]). Assume the existence of two disjoint lines $L, R$ such that $P \in\left\langle\nu_{d}(L \cup R)\right\rangle$. Take $O \in\left\langle\nu_{d}(L)\right\rangle$ and $Q \in\left\langle\nu_{d}(R)\right\rangle$ such that $P \in\langle\{O, Q\}\rangle$. Take $A \subset L$ (resp. $B \subset R$ ) be a set evincing the rak of $O$ (resp. $Q$ ) with respect to the rational normal curve $\nu_{d}(L)$ (resp. $\left.\nu_{d}(R)\right)$. Since every degree $d$ bivariate polynomial has rank $\leq 2$, we have $\sharp(A) \leq d$ and $\sharp(B) \leq d$. Since $P \in\left\langle\nu_{d}(A \cup B)\right\rangle$ and $r(P)=2 d$, we have $\sharp(A)=\sharp(B)=d$. By a theorem of Sylvester ([3, Theorem 23], [9, Theorem 4.1]) there are connected zero-dimensional schemes $A^{\prime} \subset L, B^{\prime} \subset R$ such that $\operatorname{deg}\left(A^{\prime}\right)=\operatorname{deg}\left(B^{\prime}\right)=2, O \in\left\langle\nu_{d}\left(A^{\prime}\right)\right\rangle$ and $Q \in\left\langle\nu_{d}\left(B^{\prime}\right)\right\rangle$. Since $P \in\left\langle\nu_{d}(A \cup B)\right\rangle$, the uniqueness of $Z$ gives $Z^{\prime} \sqcup\{O\}=A \cup B$, a contradiction.

Now assume that $Z$ is connected. We have $r(P)=3 d-2$ ([2, Proposition 5.19]). Since every bivariate polynomial of degree $d$ has rank at most $d$ and $r(P)>2, P \notin\left\langle\nu_{d}(E)\right\rangle$ for any union of two distinct lines.

\section{Acknowledgements}

The author was partially supported by MIUR and GNSAGA of INdAM (Italy).

\section{References}

[1] E. Ballico and J. Migliore, Smooth curves whose hyperplane section is a given set of points. Comm. Algebra 18 (1990), no. 9, 3015-3040.

[2] E. Ballico and A. Bernardi, Stratification of the fourth secant variety of Veronese variety via the symmetric rank, Adv. Pure Appl. Math. 4, no. 2, 215-250 (2013) DOI: 10.1515/apam-2013-0015

[3] A. Bernardi, A. Gimigliano, M. Idà, Computing symmetric rank for symmetric tensors, J. Symbolic. Comput. 46 (2011), no. 1, 34-53.

[4] G. Blekherman and Z. Teitler, On maximum, typical, and generic ranks, arXiv:1402.2371. 
[5] W. Buczyńska, J. Buczyński, Secant varieties to high degree veronese reembeddings, catalecticant matrices and smoothable Gorenstein schemes, J. Algebraic Geometry 23 (2014) 63-90 S 1056-3911(2013)00595-0

[6] J. Buczyński, A. Ginensky and J. M. Landsberg, Determinantal equations for secant varieties and the Eisenbud-Koh-Stillman J. London Math. Soc. (2) 88 (2013) 1-24.

[7] J. Buczyński and J. M. Landsberg, Ranks of tensors and a generalization of secant varieties, Linear Algebra Appl. 438 (2013), no. 2, 668-689.

[8] D. Eisenbud and J. Harris, Finite projective schemes in linearly general position, J. Algebraic Geom. 1 (1992), no. 1, 15-30.

[9] J. M. Landsberg and Z. Teitler, On the ranks and border ranks of symmetric tensors, Found. Comput. Math. 10 (2010), no. 3, 339-366. 